


\title{
Universal screening for meticillin-resistant Staphylococcus aureus: interim results from the NHS Scotland pathfinder project ${ }^{\text {is }}$
}

\author{
J.S. Reilly ${ }^{a, *}$, S. Stewart ${ }^{a}$, P. Christie ${ }^{b}$, G. Allardice $^{a, c}$, A. Smith $^{\text {a }}$, \\ R. Masterton ${ }^{d}$, I. Gould ${ }^{e}$, C. Williams ${ }^{f}$ \\ ${ }^{a}$ Health Protection Scotland, Glasgow, UK \\ ${ }^{\mathrm{b}}$ Quality Improvement Scotland (QIS), Edinburgh, UK \\ c University of Strathclyde, Glasgow, UK \\ ${ }^{d}$ NHS Ayrshire \& Arran Health Board, Ayrshire, UK \\ e University of Aberdeen, Aberdeen, UK \\ ${ }^{f}$ Royal Hospital for Sick Children, Glasgow, UK
}

\section{KEYWORDS}

Hospital-acquired infection;

Infection control;

Meticillin-resistant

Staphylococcus

aureus;

Universal screening

\begin{abstract}
Summary Following recommendations from a Health Technology Assessment (HTA), a prospective cohort study of meticillin-resistant Staphylococcus aureus (MRSA) screening of all admissions $(N=29690)$ to six acute hospitals in three regions in Scotland indicated that $7.5 \%$ of patients were colonised on admission to hospital. Factors associated with colonisation included re-admission, specialty of admission (highest in nephrology, care of the elderly, dermatology and vascular surgery), increasing age, and the source of admission (care home or other hospital). Three percent of all those who were identified as colonised developed hospital-associated MRSA infection, compared with only $0.1 \%$ of those not colonised. Specialties with a high rate of colonisation on admission also had higher rates of MRSA infection. Very few patients refused screening (11 patients, $0.03 \%$ ) or had treatment deferred (14 patients, $0.05 \%$ ). Several organisational issues were identified, including difficulties in achieving complete uptake of screening $(88 \%)$ or decolonisation $(41 \%)$; the latter was largely due to short duration of stay and turnaround time for test results. Patient
\end{abstract}

\footnotetext{
The full report was published on the Health Protection Scotland website on 8 April 2008: www.hps.scot.nhs.uk. ISBN 978-1-873772-27-0.

* Corresponding author. Address: Health Protection Scotland, Clifton House, 1-7 Clifton Place, Glasgow G3 7LN, UK. Tel.: +44 141 300 1100; fax: +44141300 1170 .

E-mail address: jacquelinereilly@nhs.net
} 
movement resulted in a decision to decontaminate all positive patients rather than just those in high risk specialties as proposed by the HTA. Issues also included a lack of isolation facilities to manage patients with MRSA. The study raises significant concerns about the contribution of decolonisation to reducing risks in hospital due to short duration of stay, and reinforces the central role of infection control precautions. Further study is required before the HTA model can be re-run and conclusions redrawn on the cost and clinical effectiveness of universal MRSA screening.

\section{Introduction}

Meticillin-resistant Staphylococcus aureus (MRSA) infections are associated with greater risk of treatment failure, increased patient mortality and higher costs than infections with meticillinsusceptible S. aureus. ${ }^{1,2}$ Within Europe, countries with low endemicity are observing an increase in the proportion of $S$. aureus cases due to MRSA; Scotland and the rest of the UK continue to have relatively high rates of MRSA, in common with Mediterranean countries, Romania and Ireland. ${ }^{3}$ MRSA is a particular challenge in hospitals as patients with wounds, invasive devices and weakened immune systems are at greater risk of infection than the general public. ${ }^{4}$

Uncertainty remains over the effectiveness of any single control measure compared with another, as they do not act independently and are often implemented concurrently. The Society for Healthcare Epidemiology of America guidelines have noted that multiple studies implementing surveillance, screening and contact precautions have resulted in a significant reduction in the rates of both MRSA colonisation and infection. ${ }^{5,6}$ Screening identifies patients who are either colonised or infected, which allows targeted intervention to reduce the risk of endogenous infection and transmission to others.

Universal screening of all patients for MRSA continues to be controversial. ${ }^{7,8}$ The most recent UK professional guidance on MRSA promotes targeted screening, but this has not been implemented in a consistent manner. ${ }^{9}$ Current MRSA screening practice within Scotland is locally determined, and is generally targeted on the basis of the perceived likelihood of MRSA carriage and risk of infection.

Policy proposals for universal MRSA screening of hospital patients have recently been developed in UK countries. ${ }^{10}{ }^{14}$ This process in Scotland followed the publication of the National Health Service (NHS)
Quality Improvement Scotland Health Technology Assessment (HTA) on the clinical- and cost-effectiveness of MRSA screening. ${ }^{15}$ An MRSA screening pilot study in Scotland was developed from the HTA recommendations, using the UK National Screening Committee screening criteria as a framework. These interim results present the findings from the first five months of data collection.

\section{Methods}

This was a prospective cohort study in six hospitals in three regions (NHS Ayrshire and Arran, NHS Grampian and NHS Western Isles Health Board areas) in Scotland.

Participating hospitals followed the strategy recommended by the HTA which, of the six possible strategies investigated, was judged the most costeffective and clinically effective approach. ${ }^{15}$ This strategy specified: screening all overnight admissions in acute specialties (excluding obstetrics, psychiatry and paediatrics); isolating patients identified as MRSA positive (colonised or infected); decolonising patients admitted to a specialty deemed 'high risk'; and isolating, but not decolonising, patients admitted to a 'low risk' specialty.

Each admission to hospital was considered as a single patient episode, although multiple admissions were monitored. Patients were categorised as elective (arranged) or emergency admissions.

Elective patients were screened at pre-admission clinics where possible. Patients found to be positive were provided with decolonisation therapy (mupirocin nasal ointment three times daily, and antiseptic bodywash, for five days). Elective patients who remained unscreened were screened on admission. Patients being admitted as emergencies or through inter-hospital transfer were screened on admission.

Screening staff were trained to take a nasal swab correctly and compliance was monitored 
Table I Summary demographics by Pathfinder NHS Board

\begin{tabular}{lcccc}
\hline & Ayrshire \& Arran & Grampian & Western Isles & Total \\
\hline Total patient admissions (\%) & $13700(46.1)$ & $15081(50.8)$ & $909(3.1)$ & 29690 \\
Female (\%) & $7241(53.1)$ & $7811(51.8)$ & $446(49.1)$ & $15498(52.3)^{\mathrm{b}}$ \\
Male (\%) & $6400(46.9)$ & $7262(48.2)$ & $462(50.9)$ & $14124(47.7)$ \\
Age (years), range (median) & $16-108(63)$ & $16-108(61)$ & $16-106(70)$ & $16-108(62)$ \\
Emergency admissions (\%) & $10743(78.4)$ & $8786(58.3)$ & $692(76.1)$ & $20221(68.1)$ \\
Elective admissions (\%) & $2901(21.2)$ & $6265(41.5)$ & $213(23.4)$ & $9379(31.6)$ \\
Admitted from home (\%) & $13057(96.8)$ & $13862(92.1)$ & $816(90.2)$ & $27735(94.2)$ \\
Admitted from hospital (\%) & $108(0.8)$ & $475(3.2)$ & $40(4.4)$ & $623(2.1)$ \\
Admitted from care home (\%) & $245(1.8)$ & $242(1.6)$ & $41(4.5)$ & $528(1.8)$ \\
No. of patient re-admissions (\%) & $2561(18.7)$ & $2029(13.5)$ & $171(18.8)$ & $4761(16.0)$ \\
Total patients screened (\%) & $11483(83.8)$ & $13777(91.3)$ & $900(99.0)$ & $26160(88.1)$ \\
Patients MRSA-positive on admission (\%) & $1043(7.6)$ & $1102(7.3)$ & $73(8.0)$ & $2218(7.5)$ \\
Patients with MRSA infection on admission (\%) & $39(0.28)$ & $58(0.38)$ & $1(0.11)$ & $98(0.33)$ \\
\hline NHS, National Health Service; MRSA, meticillin-resistant Staphylococcus aureus. & & \\
a Total patient admissions recorded by pathfinder auditors during the study period from 1 August until 31 December 2008. \\
$\begin{array}{l}\text { Patient capture varied by pathfinder site but was 88.1\% overall. } \\
\text { b Where numbers do not add up to total admissions, details were unknown or not recorded. }\end{array}$
\end{tabular}

throughout the study period. Any wounds or invasive device sites were also sampled using separate swabs.

Swabs were plated on to chromogenic agar, tested by latex slide test, and by a disc diffusion test for antibiotic susceptibility. All results were made available to staff on the laboratory reporting systems immediately upon confirmation by laboratory staff. Positive MRSA results were communicated directly to nurses on the wards.

MRSA-positive screens were reported to the infection control team. Average turnaround time was $48 \mathrm{~h}$ (range: 38 67). All patients who developed MRSA infection during their hospital stay were identified according to Centers for Disease Control and Prevention definitions. ${ }^{8}$ MRSA-positive patients were isolated or cohorted. With patient and clinician agreement, patients were commenced on decolonisation treatment where appropriate according to current guidelines. ${ }^{10}$
Data were analysed using Stata ${ }^{\circledR}$ software, version 9 at patient, specialty, hospital, NHS board and overall study project levels. Descriptive statistics are presented in this interim report.

\section{Results}

The demographics of patients from the six hospitals $(N=29690)$ inclusive of 15081 admissions from Grampian Health Board, 13700 from Ayrshire and Arran and 909 from Western Isles are presented in Table I.

The overall prevalence of MRSA colonisation during the study period was $7.47 \%$, representing the burden of patients requiring intervention at any given point in time. Table II shows that 26160 patients $(88.1 \%$ of total hospital admissions) were screened either at pre-admission clinics or on admission and, of these, $988(3.78 \%)$ were found to be positive for either MRSA colonisation or

Table II MRSA admission events: numbers and percentages at point of observation

\begin{tabular}{|c|c|c|c|}
\hline & Point of observation & Total no. (\%) patient admissions & $95 \% \mathrm{Cl}$ \\
\hline A. & Pre-admission screen positive & $55(0.24)$ & $0.18-0.31$ \\
\hline B. & Decolonisation pre-admission & $5(0.02)$ & $0.002-0.03$ \\
\hline C. & Overall pre-admission or admission screen positive & $988(3.78)$ & $3.52-4.03$ \\
\hline D. & Known on admission (previous positive MRSA from case notes) & $1727(5.87)$ & $5.53-6.21$ \\
\hline E. & $\begin{array}{l}\text { Total burden of MRSA positive (previous positive } \\
\text { pre-admission screen not decolonised, admission } \\
\text { screen positive, previous MRSA infection). } \\
\text { Patient admissions in more than one category have } \\
\text { not been double-counted. }\end{array}$ & $2218(7.47)$ & $7.10-7.84$ \\
\hline
\end{tabular}

MRSA, meticillin-resistant Staphylococcus aureus; $\mathrm{Cl}$, confidence interval. 
infection. Infections were most frequently skin/ soft tissue and surgical site infection. However, the total number of patients competing for isolation facilities on admission was 2218 (7.47\%), inclusive of those previously known to be MRSA positive (5.87\% of admissions). Over time, the number of known positives rose due to screening activity.

From a total of 9379 elective admissions, 2287 (24.4\%) attended pre-admission clinics. Of the 2215 screened at pre-admission, 52 (2.35\% of pre-admission screens, $0.24 \%$ of the total admission population) were found to have MRSA colonisation or infection. Only five $(9.61 \%)$ patients who were positive at pre- admission ( $0.02 \%$ of total admission population) were recorded as being successfully decolonised.

Overall, 405 out of 988 patients screening positive commenced decolonisation therapy, but only 41 patients $(4.1 \%$ of all positive screens) were successfully decolonised, and received three consecutive negative test results $48 \mathrm{~h}$ apart.

MRSA colonisation prevalence varied with specialty (Figure 1). Nephrology, care of the elderly, dermatology and vascular surgery had the highest proportion of MRSA-colonised admissions (17 20\%). Other factors influencing colonisation status on admission are described in Table III.

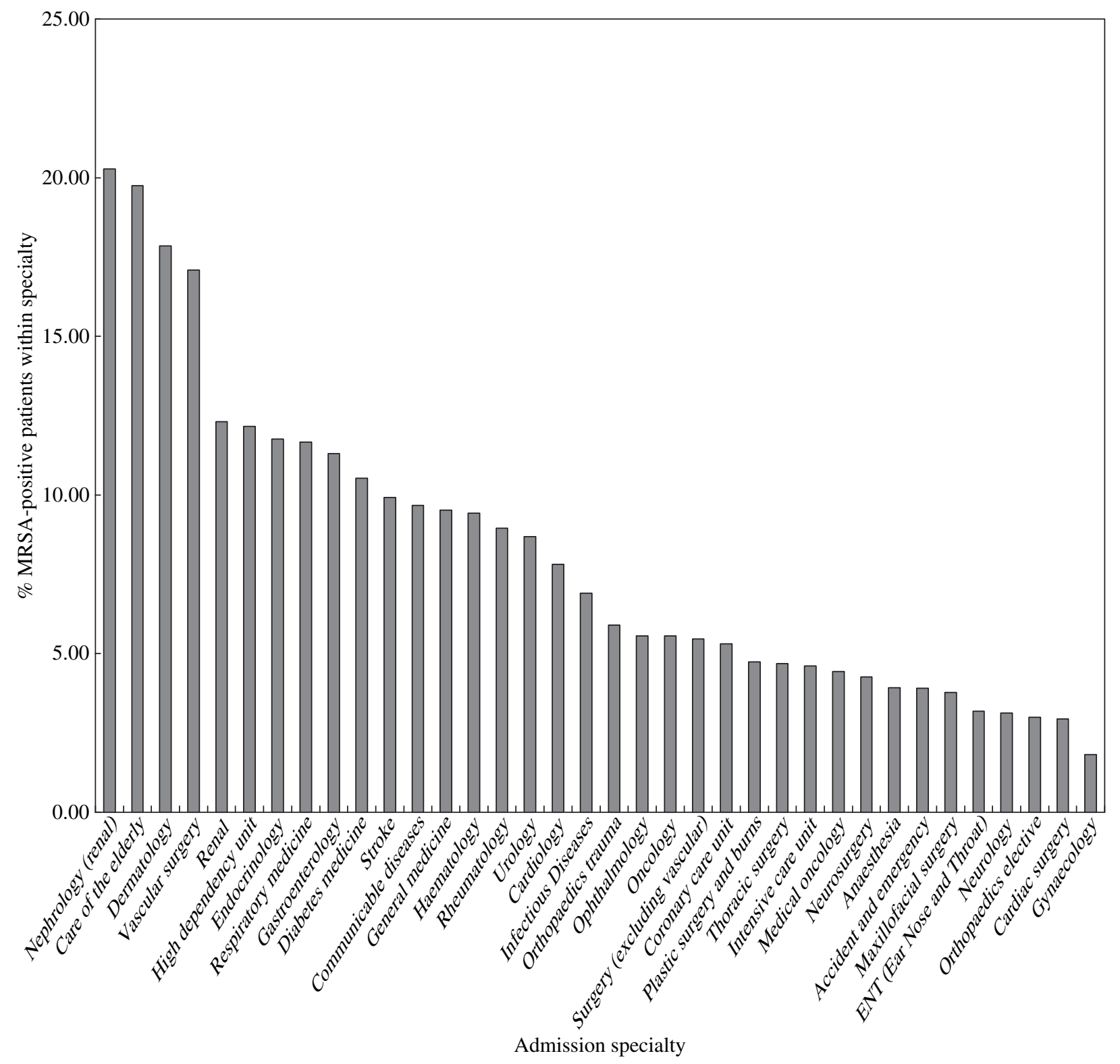

Figure 1 Proportion of patients meticillin-resistant Staphylococcus aureus positive on admission by admission specialty $(N=2184)$. 


\begin{tabular}{|c|c|c|c|}
\hline & $N$ & $\begin{array}{l}\text { Total burden } \\
\text { of MRSA + ve }\end{array}$ & $\%$ MRSA + ve \\
\hline \multicolumn{4}{|l|}{ Age } \\
\hline$<65$ years & 16765 & 726 & 4.3 \\
\hline$>65$ years & 12922 & 1490 & 11.5 \\
\hline \multicolumn{4}{|l|}{ Gender } \\
\hline Male & 14124 & 1122 & 7.9 \\
\hline Female & 15498 & 1090 & 7.0 \\
\hline \multicolumn{4}{|l|}{ Admission type } \\
\hline Elective & 9379 & 478 & 5.1 \\
\hline Emergency & 20221 & 1732 & 8.6 \\
\hline \multicolumn{4}{|l|}{ Admitted from } \\
\hline Home & 27735 & 1913 & 6.9 \\
\hline Care home & 528 & 194 & 36.7 \\
\hline Hospital & 623 & 79 & 12.7 \\
\hline \multicolumn{4}{|l|}{ Admission events } \\
\hline First admission & 24929 & 1620 & 6.5 \\
\hline Repeat admission & 4761 & 598 & 12.6 \\
\hline
\end{tabular}

The MRSA infection incidence during the study was $0.3 \%$ (97 admissions). The highest MRSA infection rates were in anaesthesia $(2.94 \%)$, nephrology $(2.04 \%)$, diabetes medicine $(1.75 \%)$ and vascular surgery $(1.53 \%)$. Less than a tenth $(7.5 \%)$ of all infections were bloodstream infections. Three percent of those who developed healthcare-associated (HA) MRSA infection were positive at admission or pre-admission clinics, compared with $0.1 \%$ who screened negative.

\section{Discussion}

This is the first UK pilot study for a national MRSA screening programme. The total prevalence of MRSA colonisation at time of admission was found to be $7.5 \%$. The percentage of patients identified as colonised by screening was $3.8 \%$ but, in addition, $5.9 \%$ of admissions (screened or not) were known previous MRSA positives. Prevalence of MRSA colonisation is defined in different ways in the published literature, which makes comparisons difficult but highlights the need for consistency. ${ }^{1,8,16}$ Studies published since the HTA have indicated an observed colonisation prevalence of $5.1 \%$ in all patients in a Swiss teaching hospital, $6.7 \%$ in a study of emergency patients and $5.1 \%$ in surgical patients in the UK. $8,16,17$

Higher age, particular clinical specialties, emergency admission, admission from other hospitals or care homes, and a history of multiple admissions were all found to be associated with higher risks of MRSA colonisation.

Patients aged $>65$ years were more likely to be colonised on admission to hospital $(11.5 \% \geq 65$ years vs $4.4 \%<65$ years); there was no statistically significant difference between males and females. These findings are consistent with other reports. ${ }^{1,18} 21$ Colonisation prevalence also varied by hospital (6 9\%) and by specialty (0 20.3\%). The specialties with the highest prevalences (17 20\%) were nephrology, care of the elderly, dermatology and vascular surgery. Caution must be used in interpreting these figures, however, as the higher risk specialties generally have longer durations of stay, and some additional cases may have been excluded at this interim stage in our study. Nonetheless, the colonisation burden of one in five patients indicates that these are specialties which are priority areas for screening, despite an absence of evidence for universal screening.

Colonisation prevalence varied between elective patients $(2.4 \%$ of all pre-admission screens) and emergency admissions (4.3\% all emergency patients screened on admission). However, only a quarter of elective admissions in the study $\mathbf{( 7 . 5 \%}$ of all admissions) were screened at pre-admission clinics; of these, $2.35 \%$ were found to be colonised and only a small proportion of these patients (9.6\%) were successfully decolonised before admission. The observed uptake $(97 \%)$ and ease of capturing these patients is therefore counterbalanced by the low yield of positives identified and decolonised, as well as the low colonisation rate and the small proportion of total patients in this group. It proved challenging to capture patients seen pre-admission at general outpatient clinics. Any added benefit of pre-admission screening is also affected by the timing of clinics, relative to decolonising and re-testing prior to admission.

Previous MRSA colonisation is a recognised risk factor for an MRSA-positive screen, and was found in $5.9 \%$ of admissions in the study. Admission from care homes or other hospitals has been associated with higher colonisation risk, confirmed by the current study $\left(25 \%\right.$ and $7.5 \%$ respectively). ${ }^{22,23}$ However, transfers from care homes or other hospitals represented only a small proportion (3.8\%) of all admissions. In line with the HTA assumption, the proportion of patients with known MRSA status increased over the study period, due to increasing ascertainment in patients with multiple admissions which, of itself, may be a risk factor for colonisation. This raises the issue of continuing decolonisation regimens post discharge, for which there is no current national guidance, in order to minimise the 
risk of persistent colonisation on re-admission. During the five months of the study, $16 \%$ of total admissions were re-admissions and almost onethird of all MRSA-positive admissions were readmissions.

This interim analysis does not examine all risk factors for colonisation and, as such, cannot generate a full clinical risk assessment (CRA). The potential importance of CRA is supported by our observation that almost three-quarters of those patients admitted who were pre-emptively isolated on the basis of CRA were found to be positive thereafter by nasal screening. This observation varied by healthcare board within the study, which may reflect the variation in CRA tools used.

The incidence of MRSA infections was 0.3\% (109 infections in 98 patients). Around half were HA MRSA and half were community-acquired MRSA. This is broadly consistent with other reports. ${ }^{17}$ Skin and soft tissue infections were the most common type of infection, followed by surgical infections. It should be noted that the observed rates are likely to be an underestimate of infection rates as some long-stay patients may have been excluded from the study as they remained non-discharged, and post-discharge infections were not within the scope of the study. Three percent of those who developed HA MRSA infection were colonised on admission, compared with $0.1 \%$ of those screened negative on admission. Patients in anaesthetics, nephrology, diabetes medicine and vascular surgery specialties were more likely to develop MRSA infections, being specialties with patient case-mix and interventions associated with increased risk of MRSA.

The screening programme in the study hospitals to date resulted in very few patient refusals for screening (11 patients, $0.03 \%$ ) or treatment deferrals (14 patients, $0.05 \%$ ). However, a number of practical organisational issues were identified. Patient movement within the hospital between low and high risk specialty wards made selective decolonisation a challenge and a decision was taken to decolonise all MRSA positive patients. Organisational issues also included a lack of single room facilities to manage patients with MRSA. The HTA assumed 3 isolation rooms per 25 bed ward for MRSA patients. This study found an uneven distribution of rooms between wards and competing priorities for the use of these rooms. Most patients (78\%) were cohorted or separated from other patients during their stay, a practice which is widespread but carries little supporting evidence of effectiveness, and some were managed on open wards. This reinforces the crucial importance of all staff using Standard Infection Control Precautions at all times for all patients.
Operational issues also included difficulties in achieving full uptake of screening ( $88 \%$ achieved), and compliance with decolonisation (only $41 \%$ of MRSA-positive patients had decolonisation initiated). The latter was mostly due to short durations of admission and minimum two day turnaround time for obtaining screening results from the laboratory. ${ }^{24}$ Only $4.1 \%$ of known MRSA positives were documented as having been successfully decolonised. These findings, in combination with false negative test results and the relatively low effectiveness of current decolonisation techniques raise significant concerns about the small proportion of colonised patients actually being rendered MRSA-negative, both in terms of staffing and financial resource use and, critically, the real reduction in risk achieved. ${ }^{15}$

\section{Conflict of interest statement None declared.}

\section{Funding source}

This study was funded by the Scottish Government Health Directorate.

\section{References}

1. Ritchie K, Craig J, Eastgate J, et al. The clinical and cost effectiveness of screening for meticillin-resistant Staphylococcus aureus (MRSA). Edinburgh: NHS QIS; 2007.

2. Wyllie DH, Peto TE, Crook D. MRSA bacteraemia in patients on arrival in hospital: a cohort study in Oxfordshire 19972003. Br Med J 2005; 29;331(7523):992.

3. Blot SI, Vandewoude KH, Hoste EA, Colardyn FA. Outcome and attributable mortality in critically ill patients with bacteremia involving methicillin-susceptible and methicillinresistant Staphylococcus aureus. Arch Intern Med 2002; 162:2229-2235.

4. European Antimicrobial Resistance Surveillance System. Annual report. Bilthoven: European Centre for Disease Prevention and Control; 2006.

5. Selvey LA, Whitby M, Johnson B. Nosocomial methicillin-resistant Staphylococcus aureus bacteremia: is it any worse than nosocomial methicillin-sensitive Staphylococcus aureus bacteraemia? Infect Control Hosp Epidemiol 2000;21:645-648.

6. Cooper BS, Stone SP, Kibbler CC, et al. Systematic review of isolation policies in the hospital management of methicillinresistant Staphylococcus aureus: a review of the literature with epidemiological and economic modelling. Health Technol Assess 2003;7:1-194.

7. Muto CA, Jernigan JA, Ostrowsky BE, et al. SHEA guideline for preventing nosocomial transmission of multidrug-resistant strains of Staphylococcus aureus and enterococcus. Infect Control Hosp Epidemiol 2003;24:362-386.

8. Harbarth S, Fankhauser C, Schrenzel J, et al. Universal screening for methicillin-resistant Staphylococcus aureus at hospital admission and nosocomial infection in surgical patients. J Am Med Assoc 2008;299:1149-1157. 
9. Robicsek A, Beaumont JL, Paule SM, et al. Universal surveillance for methicillin-resistant Staphylococcus aureus in 3 affiliated hospitals. Ann Intern Med 2008;148:409-418.

10. Coia JE, Duckworth GJ, Edwards DI, et al. Guidelines for the control and prevention of meticillin-resistant Staphylococcus aureus (MRSA) in healthcare facilities. J Hosp Infect 2006;63(Suppl 1):S1-44.

11. Martin P. New funding for the National MRSA Screening Programme (CEL 55 2008). Edinburgh: Scottish Government; 2008.

12. Chief Medical Officer/Chief Nursing Officer. MRSA Screening CNO (2008) 02; CMO (2008) 02. Cardiff: Welsh Assembly Government; 2008.

13. McBride M. Best practice guidance on screening for MRSA colonisation. Belfast: Northern Ireland Government; 2008.

14. Department of Health. MRSA screening - operational guidance. London: DoH; 2008.

15. Centers for Disease Control and Prevention. NNIS manual. Atlanta: CDC; 1999.

16. Rao G, Michalczyk P, Nayeem N, Walker G, Wigmore L. Prevalence and risk factors for meticillin-resistant Staphylococcus aureus in adult emergency admissions - a case for screening all patients? J Hosp Infect 2007;66:15-21.

17. Harbarth S, Sax H, Uckay I, et al. A predictive model for identifying surgical patients at risk of methicillin-resistant Staphylococcus aureus carriage on admission. J Am Coll Surg 2008;207:683-689.

18. Cosgrove SE, Qi $\mathrm{Y}$, Kaye KS, Harbarth S, Karchmer AW, Carmeli $Y$. The impact of methicillin resistance in
Staphylococcus aureus bacteremia on patient outcomes: mortality, length of stay, and hospital charges. Infect Control Hosp Epidemiol 2005;26:166-174.

19. Lucet JC, Paoletti X, Lolom I, et al. Successful long-term program for controlling methicillin-resistant Staphylococcus aureus in intensive care units. Intensive Care Med 2005;31: 1051-1057.

20. Vovko P, Retelj M, Cretnik TZ, et al. Risk factors for colonization with methicillin-resistant Staphylococcus aureus in a long-term-care facility in Slovenia. Infect Control Hosp Epidemiol 2005;26:191-195.

21. Khurram IM, Khan SA, Khwaja AA, et al. Risk factors for clinical infection in patients colonized with methicillin resistant Staphylococcus aureus (MRSA). J Pak Med Assoc 2004;54:408-412.

22. Hidron Al, Kourbatova EV, Halvosa JS, et al. Risk factors for colonization with methicillin-resistant Staphylococcus aureus (MRSA) in patients admitted to an urban hospital: emergence of community-associated MRSA nasal carriage. Clin Infect Dis 2005;41:159-166.

23. Haley CC, Mittal D, Laviolette A, Jannapureddy S, Parvez N, Haley RW. Methicillin-resistant Staphylococcus aureus infection or colonization present at hospital admission: multivariable risk factor screening to increase efficiency of surveillance culturing. J Clin Microbiol 2007;45: 3031-3038.

24. Health Protection Scotland. National Services Scotland, NHS Scotland. MRSA Screening Pathfinder Programme Interim Report 2009. Glasgow: NSS HPS; 2009. 